\title{
Two types of modes in finite size one-dimensional coaxial photonic crystals: General rules and experimental evidence
}

\author{
E. H. El Boudouti, ${ }^{1} *$ Y. El Hassouani, ${ }^{1}$ B. Djafari-Rouhani, ${ }^{2}$ and H. Aynaou ${ }^{1}$ \\ ${ }^{1}$ Laboratoire de Dynamique et d'Optique des Matériaux, Département de Physique, Faculté des Sciences, \\ Université Mohamed I, Oujda, Morocco \\ ${ }^{2}$ Institut d'Electronique, de Microélectronique et de Nanotechnologie (IEMN), UMR CNRS 8520, UFR de Physique, \\ Université des Sciences et Technologies de Lille, 59655 Villeneuve d'Ascq, France
}

(Received 25 April 2007; published 29 August 2007)

\begin{abstract}
We demonstrate analytically and experimentally the existence and behavior of two types of modes in finite size one-dimensional coaxial photonic crystals made of $N$ cells with vanishing magnetic field on both sides. We highlight the existence of $N-1$ confined modes in each band and one mode by gap associated to either one or the other of the two surfaces surrounding the structure. The latter modes are independent of $N$. These results generalize our previous findings on the existence of surface modes in two semi-infinite superlattices obtained from the cleavage of an infinite superlattice between two cells. The analytical results are obtained by means of the Green's function method, whereas the experiments are carried out using coaxial cables in the radiofrequency regime.
\end{abstract}

DOI: 10.1103/PhysRevE.76.026607

PACS number(s): 41.20.Jb, 77.84.Lf

\section{INTRODUCTION}

The problem of propagation of electromagnetic waves in artificial periodic dielectric materials received a great deal of attention in the last two decades $[1,2]$. Of particular interest is the existence of photonic band gaps in the electromagnetic band structures of such materials called photonic crystals (PCs). These structures present unusual properties which can be exploited in the control and the guidance of the propagation of light $[3,4]$. However, in real materials, the PCs are often of finite size with free surfaces. The study of electromagnetic wave propagation in finite size one-dimensional (1D) multilayer PCs (or superlattice) was largely developed theoretically and experimentally [5-17]. In general, the finite size structure is deposited on a homogeneous substrate with or without a buffer layer or encapsulated with a cap layer [9]. The results obtained show that, in addition to the standing waves in the finite size structure, there exists additional modes inside the band gaps induced by the different inhomogeneities introduced in the periodic structure.

Some years ago [18] we demonstrated that in the case of transverse elastic waves, the creation of two semi-infinite SLs from the cleavage of an infinite $N$-layer SL gives rise to one surface mode by gap for any value of the wave vector parallel to the interfaces. This mode belongs to one or the other of the two complementary SLs. These results were confirmed experimentally in a two-layer elastic SL $[19,20]$. Recently $[21,22]$, we have given theoretical demonstration and experimental evidence that the same conclusions hold in quasi-one-dimensional (Q1D) periodic systems made of coaxial cables with different geometries such as comblike [21] and serial loop structures [22]. The analogy between surface elastic waves in multilayered structures and surface electromagnetic waves in coaxial cables is straightforward in two limiting cases, namely, when the boundary conditions at the ends of the coaxial cables are either $E=0$ or $H=0$. Let us

\footnotetext{
*Corresponding author. elboudouti@yahoo.fr
}

recall that these two conditions mean, respectively, that the two constituting conductors of the coaxial cables are, or are not, short circuited. However, this analogy is not fulfilled in the case of multilayered optical structures where the boundary conditions at the surface are neither $E=0$ nor $H=0$. Indeed, when dealing with layered media, the SL is in contact with a dielectric homogeneous medium (such as vacuum, for example) and therefore, the continuity of the transverse component of $H$ and the normal component of the displacement field $D$ should be satisfied at the surface. For these reasons, coaxial cables are good candidates for highlighting general rules about confined and surface electromagnetic modes in finite size 1D structures in the above mentioned cases (i.e., $E=0$ or $H=0$ ). Of course, these rules do not apply for optical multilayered media [11]. Also, it was shown that coaxial cables present an easily realizable experimental approach to the study of wave interference phenomena such as band gap structures with or without defect modes [23-25], superluminal and subluminal effects [25-27], and standing waves [21,22].

In this paper, we consider a finite size SL made of $\mathrm{N}$ unit cells [Figs. 1(a) and 1(b)]. The left and right surfaces of one unit cell [Fig. 1(a)], indicated by a circle and a cross, are in general different. We shall call them complementary surfaces. We suppose that the boundary conditions at both ends of the finite structure [Fig. 1(b)] are of type $H=0$. Our goal is to show analytically and experimentally the existence of $N$ -1 modes in each allowed band and one additional mode by gap induced by one of the two complementary surfaces surrounding the structure. We show that these modes are those of a unique single cell (i.e., $N=1$ ). In the particular case where the cells are symmetrical (i.e., with the same surface terminations), we show that the surface modes fall at the band edges.

Contrary to the usual results where the defect modes induced by the two surfaces surrounding the finite structure depend strongly on its size $[9,17]$, we show that the modes induced by the two complementary surfaces of the finite structure [Fig. 1(b)] are independent of $N$ and coincide ex- 
actly with the surface modes associated to two complementary semi-infinite SLs [Fig. 1(c)] obtained from the cleavage of an infinite SL [Fig. 1(d)] [18,21,22]. So, even the standing waves of only one cell $[N=1$, Fig. $1(\mathrm{a})]$ give the surface modes of two complementary SLs [Fig. 1(c)]. Among the different theoretical models, the transfer-matrix [28] and Green's function methods $[29,30]$ are quite suitable to deduce the eigenmodes and eigenvectors as well as the transmission and reflection coefficients in composite media. In addition to these quantities, the Green's function approach also enables one to deduce easily the local and total densities of states $[9,11,17,18,22]$. The experiments are realized on PCs based on coaxial BNC connectors with different impedances called coaxial PCs. The propagation in these structures is monomode [30] and one can obtain very accurate experimental results that may be fitted with a simple 1D theoretical model. The interference of the multiple reflected waves leads to the same phenomena in the radiofrequency range as for light propagation through a photonic crystal [31-33].

It is worth noting that different results (eigenmodes and spatial localization) are obtained if the boundary conditions on both ends of the finite size structure [Fig. 1(b)] are of type $E=0$ instead of $H=0$ [21]. However, the same rules apply to confined and surface modes in these structures. For the sake of brevity, we shall avoid giving the results concerning the case $E=0$. Let us mention that similar results to those presented here are found theoretically by Ren $[34,35]$ for the complete confinement of electronic states in 1D crystals of finite size.

This paper is organized as follows. In Sec. II, we give a short summary of the theoretical model and the main analytical results. Section III gives the principal numerical and experimental results about confined and surface modes in finite size coaxial PCs with symmetric and asymmetric cells. Conclusions are presented in Sec. IV.

\section{METHOD OF THEORETICAL AND NUMERICAL CALCULATION}

\section{A. Interface response theory of continuous media}

Our theoretical analysis is performed with the help of the interface response theory of continuous media, which allows the calculation of the Green's function of any composite material. In what follows, we present the basic concept and the fundamental equations of this theory [29]. Let us consider any composite material contained in its space of definition $D$ and formed out of $N$ different homogeneous pieces located in their domains $D_{i}$. Each piece is bounded by an interface $M_{i}$, adjacent in general to $j(1 \leqslant j \leqslant J)$ other pieces through subinterface domains $M_{i j}$. The ensemble of all these interface spaces $M_{i}$ will be called the interface space $M$ of the composite material. The elements of the Green's function $g(D D)$ of any composite material can be obtained from [29]

$$
\begin{aligned}
g(D D)= & G(D D)-G(D M) G^{-1}(M M) G(M D) \\
& +G(D M) G^{-1}(M M) g(M M) G^{-1}(M M) G(M D),
\end{aligned}
$$

where $G(D D)$ is the reference Green's function formed out (a)

$$
0^{0-1}
$$

(b)

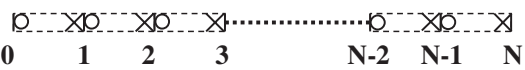

(c)

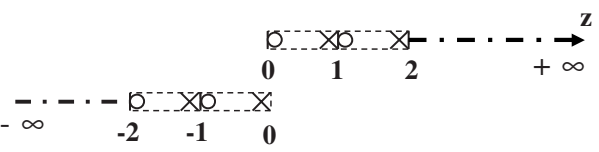

(d) $\begin{array}{ccccccc}-\infty & - & - & & & & \\ -\infty & -2 & -1 & 0 & 1 & 2 & +\infty\end{array}$

FIG. 1. Schematic representation of (a) a finite cell bounded by the space of interfaces $M=\{0,1\}$, the circle and the cross indicate the left and the right surfaces of the cell, respectively. (b) A finite SL constituted of $N$ cells. (c) Two semi-infinite SLs obtained from the cleavage of an infinite SL (d) between two cells. Notice the similarities between the surfaces ending the two complementary SLs $[(c)]$ and those corresponding to a finite SL $[(a),(b)]$.

of truncated pieces in $D_{i}$ of the bulk Green's functions of the infinite continuous media and $g(M M)$ the interface element of the Green's function of the composite system. The knowledge of the inverse of $g(M M)$ is sufficient to calculate the interface states of a composite system through the relation [29]

$$
\operatorname{det}\left[g^{-1}(M M)\right]=0 .
$$

Moreover if $U(D)$ represents an eigenvector of the reference system, Eq. (1) enables the calculation of the eigenvectors $u(D)$ of the composite material [29]

$$
\begin{aligned}
u(D)= & U(D)-U(M) G^{-1}(M M) G(M D) \\
& +U(M) G^{-1}(M M) g(M M) G^{-1}(M M) G(M D) .
\end{aligned}
$$

In Eq. (3), $U(D), U(M)$, and $u(D)$ are row vectors. Equation (3) provides a description of all the waves reflected and transmitted by the interfaces, as well as the reflection and transmission coefficients of the composite system. In this case, $U(D)$ is a bulk wave launched in one homogeneous piece of the composite material [30].

\section{B. Dispersion relations of infinite, finite, and semi-infinite periodic 1D structures}

Consider an infinite SL made of a periodic repetition of a given 1D cell [Fig. 1(d)]. In this work, the cell is a multiwaveguide one-dimensional system (see below). Using the Green's function formalism, each cell is characterized by a $2 \times 2$ matrix constituted by the Green's function elements on the surfaces bounding the cell [Fig. 1(a)]. The boundary conditions on both sides of the cell are $H=0$ (vanishing magnetic field). The inverse of the $2 \times 2$ matrix can be written explicitly as

$$
[g(M M)]^{-1}=\left(\begin{array}{ll}
a & b \\
b & c
\end{array}\right),
$$

where the space of interface $M=\{0,1\}$. The four matrix elements are real quantities functions of the different parameters 
of the constituents of the cell (see below). It is worth noting that in general, $a \neq c$; however, if the cell is symmetrical (i.e., with the same surface terminations) then $a=c$. The eigenmodes of the elementary cell are given by Eq. (2), namely,

$$
a c-b^{2}=0 .
$$

The Green's function of the infinite SL made of a periodic repetition of a given cell [Fig. 1(d)] is obtained by a linear juxtaposition of the $2 \times 2$ matrices [Eq. (4)] in the interface domain of all the sites $n$. We obtain a tridiagonal matrix where the diagonal and off-diagonal elements of this matrix are given, respectively, by $a+c$ and $b$.

Taking advantage of the translational periodicity of this system along the $z$ axis, this matrix can be Fourier transformed as [18]

$$
[g(k, M M)]^{-1}=2 b[\cos (k D)-\xi],
$$

where $k$ is the modulus of the one-dimensional reciprocal vector (Bloch wave vector), $D$ is the period of the SL, and $\xi=-(a+c) / 2 b$.

The dispersion relation of the infinite periodic SL [Fig. 1(d)] is given by Eqs. (2) and (6), namely,

$$
\cos (k D)=-(a+c) / 2 b .
$$

On the other hand, in the $k$ space, the surface Green's function is

$$
[g(k, M M)]=\frac{1}{2 b[\cos (k D)-\xi]} .
$$

After inverse Fourier transformation, Eq. (8) gives

$$
g\left(n, n^{\prime}\right)=\frac{1}{b} \frac{t^{\left|n-n^{\prime}\right|+1}}{t^{2}-1},
$$

where $n$ and $n^{\prime}$ denote the positions of the different interfaces between the cells and $t=e^{i k D}$.

Consider now a finite SL bounded by the two surfaces $n$ $=0$ and $n=N$ [Fig. 1(b)] with vanishing magnetic field on both ends. Following the same calculation procedure as in our previous works on acoustic waves in finite SLs [36,37], the $2 \times 2$ inverse matrix in the space of interface $M^{\prime}$ $=\{0, N\}$ of the finite SL can be written as

$$
\left[g\left(M^{\prime} M^{\prime}\right)\right]^{-1}=\left(\begin{array}{ll}
a^{\prime} & b^{\prime} \\
b^{\prime} & c^{\prime}
\end{array}\right),
$$

where

$$
\begin{gathered}
a^{\prime}=a+b t-t^{2 N}\left(a+\frac{b}{t}\right), \\
b^{\prime}=-b t^{N}\left(t-\frac{1}{t}\right),
\end{gathered}
$$

and

$$
c^{\prime}=-a-\frac{b}{t}+t^{2 N}(a+b t) .
$$

The eigenmodes of the finite SL are given by Eqs. (2) and (10), namely, $a^{\prime} c^{\prime}-b^{\prime 2}=0$ or, equivalently,

$$
\left(t+\frac{b}{a}\right)\left(t+\frac{a}{b}\right)\left(1-t^{2 N}\right)=0 .
$$

Now, if the finite composite system is sandwiched (grafted) horizontally (vertically) between two homogeneous waveguides characterized by their impedance $Z_{s}$ [see the insets of Figs. 2(c) and 2(e)], then an incident plane wave launched from the left waveguide gives rise to the transmission functions in the right waveguide as [22]

$$
\operatorname{tr}_{h}=\frac{2 j b^{\prime} / Z_{s}}{a^{\prime} c^{\prime}-b^{\prime 2}-\left(1 / Z_{s}\right)^{2}-j\left(a^{\prime}+c^{\prime}\right) / Z_{s}}
$$

and

$$
\operatorname{tr}_{v}=\frac{-2 j c^{\prime} / Z_{s}}{a^{\prime} c^{\prime}-b^{\prime 2}-2 j c^{\prime} / Z_{s}},
$$

respectively, where $h$ and $v$ stand for horizontal and vertical insertion of the finite PC. $a^{\prime}, b^{\prime}$, and $c^{\prime}$ are given by Eqs. (11)-(13), respectively. The transmission function can be written in an explicit complex form as $\operatorname{tr}=\alpha+j \beta=|\operatorname{tr}| e^{j \varphi}$, where $|\operatorname{tr}|$ is the transmission coefficient, $\varphi$ $=\arctan (\beta / \alpha) \pm m \pi$ is the phase associated with the transmission field, and $m$ is an integer.

\section{General rules about confined and surface modes}

As it is expected, we should have $N$ eigenmodes in each band gap. However, Eq. (14) shows that there are two types of eigenmodes in the finite structure.

(1) If the wave vector $k$ is real which corresponds to an allowed band, then the eigenmodes of the finite SL are given by the third term in Eq. (14), namely,

$$
\sin (N k D)=0,
$$

which gives

$$
k D=\frac{m \pi}{N}, \quad m=1,2, \ldots, N-1,
$$

whereas the first and second terms in Eq. (14) cannot vanish in the bulk bands.

(2) If the wave vector $k$ is imaginary (modulo $\pi$ ) which corresponds to a forbidden band, then the eigenmodes are given by the two first terms of Eq. (14), namely,

$$
t=-\frac{a}{b}
$$

and

$$
t=-\frac{b}{a},
$$

whereas the third term in Eq. (14) cannot vanish inside the gap since $t$ should satisfy the condition

$$
|t|<1
$$

to ensure the decaying of surface states from the surface. 

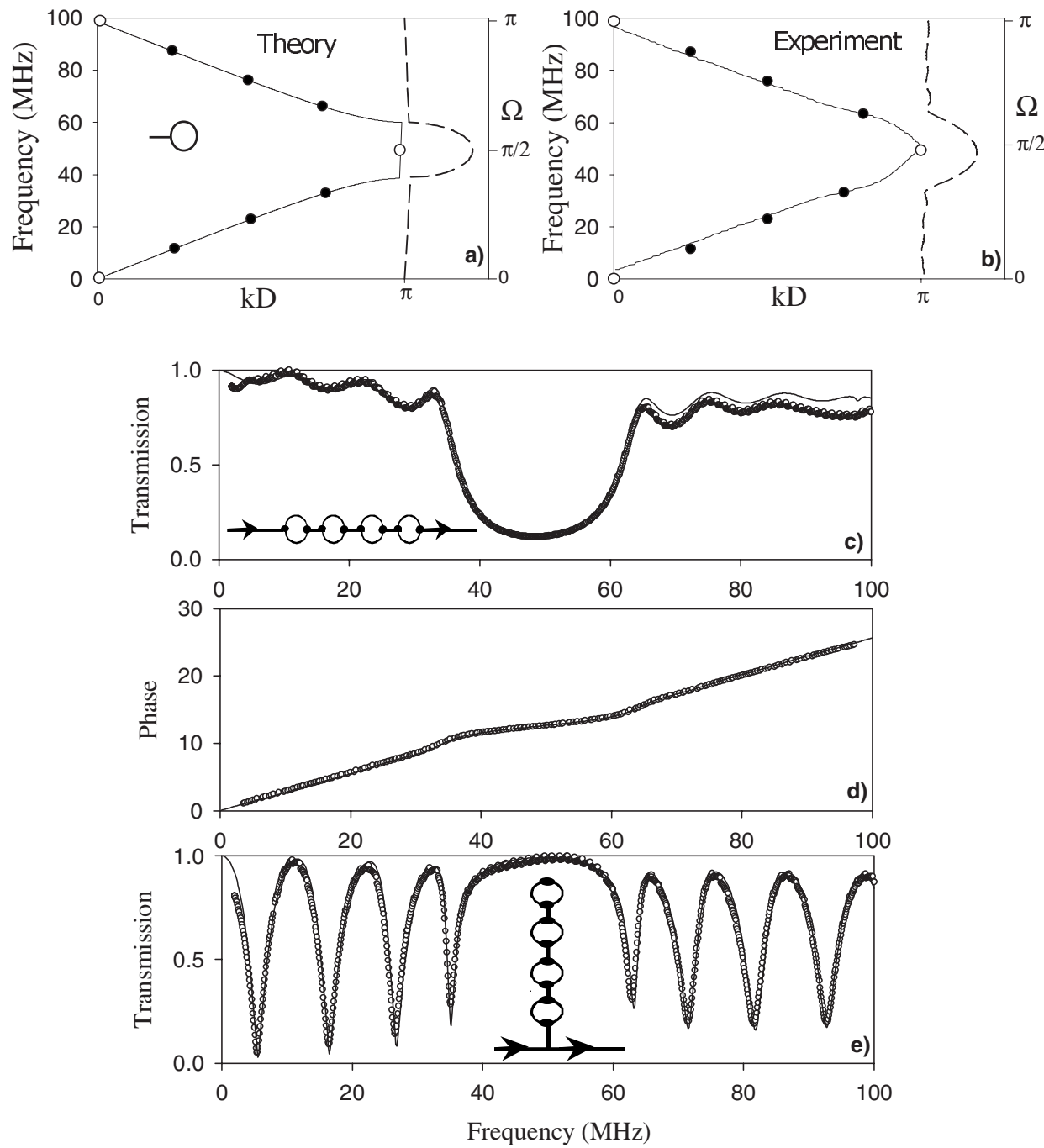

FIG. 2. (a) Theoretical band structure of an infinite SL. Each cell is made of a standard coaxial cable of length $d_{1}=1 \mathrm{~m}$ and impedance $Z=50 \Omega$ connected to a symmetric loop made of two identical coaxial cables of length $d_{2}=1 \mathrm{~m}$ and impedance $Z=50 \Omega$ (see the inset). Solid and open circles correspond to the eigenmodes of a finite structure made of $N=4$ cells. (b) Same as (a) but for the experimental results. (c) and (d) represent, respectively, theoretical (solid line) and experimental (open circles) variations of the transmission amplitude and phase through a finite SL containing $N=4$ cells [see the inset of (c)] with the same characteristic lengths as in (a). (e) Same as (c) but for the finite structure grafted vertically along the guide (see the inset).

In addition, we remark that if $N \rightarrow \infty$ the term $t^{2 N}$ vanishes and therefore the two expressions [Eqs. (19) and (20)] give the surface modes for two semi-infinite SLs with complementary surfaces [Fig. 1(c)].

Equations (19) and (20) can be written in a unique explicit form by replacing them in Eq. (7) and factorizing by the factor $\frac{1}{b}$, one obtains

$$
a c-b^{2}=0 .
$$

Therefore, the surface modes of one semi-infinite SL are given by Eq. (22) together with the condition $\left|\frac{a}{b}\right|<1$ [Eqs. (19) and (21)], whereas the surface modes of the complementary SL are given by Eq. (22) but with the condition $\left|\frac{b}{a}\right|<1$ [Eqs. (20) and (21)]. This result shows that if a surface mode appears on the surface of one SL, it does not appear on the other surface of the complementary SL. More- over, Eq. (22) shows that the expression giving the surface modes for two complementary SLs is exactly the same expression giving the eigenmodes of one cell [Eq. (5)].

In addition to these results, let us recall briefly another result concerning the existence and behavior of surface waves in a quasi-one-dimensional SL [22], namely, the creation from the infinite SL of a surface with vanishing magnetic field gives rise to $\delta$ peaks of weight $(-1 / 4)$ in the density of states, at the edges of the SL bulk bands. Now, one considers together the two complementary SLs: (i) the variation of density of states presents a loss of one half-mode at the limit of each band, i.e., one mode by band and (ii) the variation of density of states vanishes inside the bulk bands of the two complementary SLs. These two results associated with the necessary conservation of the total number of modes show that one surface mode of weight unity by gap must exist to compensate the loss of one mode by band. These 
modes are associated with either one or the other surface of the two SLs.

From all the above results, one can deduce that a finite SL constituted of $N$ cells gives rise to $N-1$ confined modes inside the bulk bands of the SL and one surface mode in each gap of the SL that may be attributed to one of the two surfaces surrounding the finite SL. The surface modes are independent of $N$ and coincide with those of two semi-infinite SLs obtained by the cleavage of an infinite SL between two cells.

In the particular case of a symmetric cell, the surface modes inside the gaps move to the band gap edges. Indeed, in symmetric cells, $a=c$ and therefore Eq. (22) reduces to $a= \pm b$. This expression corresponds to a band gap edge as $\cos (k d)= \pm 1$ [Eq. (7)]. In what follows, we shall give a numerical and experimental confirmation of these results in the case of electromagnetic wave propagation in coaxial cables treated as quasi-one-dimensional waveguides.

\section{NUMERICAL AND EXPERIMENTAL RESULTS}

\section{A. Case of a finite periodic structure made of asymmetric cells}

In what follows, we consider a finite periodic photonic crystal where each cell is made of a standard coaxial cable of length $d_{A}=1 \mathrm{~m}$ and impedance $Z=50 \Omega$ and a loop made of two identical coaxial cables of length $d_{B}=1 \mathrm{~m}$ and impedance $Z=50 \Omega$ [see the inset of Fig. 2(a)]. We can show easily (see Ref. [25]) that the loop is equivalent to a coaxial cable of length $d_{B}$ and half the impedance of the constituting cables (i.e., $Z=25 \Omega$ ). Therefore, we shall call $Z_{A}=50 \Omega$ and $Z_{B}=25 \Omega$ the impedances of the segment and the loop, respectively. Each cell becomes equivalent to a $50 \Omega / 25 \Omega$ bisegment. One can notice that the two surfaces surrounding the cell are different and therefore the cell is asymmetric.

When applied to a SL made of a periodic repetition of a segment and a loop, the general dispersion relation [Eq. (7)] gives the well-known relation

$$
\cos (k D)=C_{A} C_{B}-0.5\left(Z_{A} / Z_{B}+Z_{B} / Z_{A}\right) S_{A} S_{B},
$$

where $C_{A, B}=\cos \left(\omega d_{A, B} \sqrt{\varepsilon} / c\right), S_{A, B}=\sin \left(\omega d_{A, B} \sqrt{\varepsilon} / c\right)$, and $D$ $=d_{A}+d_{B}$.

In the particular case where $d=d_{A}=d_{B}=1 \mathrm{~m}$ and $Z_{A} / Z_{B}$ $=2$, the above equation becomes simply

$$
\cos (k D)=1-\frac{9}{4} \sin ^{2}(\Omega),
$$

where $\Omega=\omega d \sqrt{\varepsilon} / c$ is the reduced frequency.

The limits of the band gaps are given by the successive sequences $k D=0, \pi, \pi, 0,0, \pi, \pi, 0, \ldots$, and therefore $\Omega$ $\simeq 0,0.39 \pi, 0.61 \pi, \pi, \pi, 1.39 \pi, 1.61 \pi, 2 \pi, \ldots$.

These results show that the band gap structure is periodic every $\Omega=\pi$. The width of the successive bands is about $0.4 \pi$, the width of the gaps at the edge of the Brillouin zone is about $0.2 \pi$, whereas the width of the gaps at the center of the Brillouin zone vanishes. These results are confirmed theoretically [Fig. 2(a)] and experimentally [Fig. 2(b)], where we have plotted the dispersion curves (frequency versus $k D$ ) for the periodic structure depicted above. The ex- perimental curves are obtained from the amplitude $|t|$ [Fig. 2(c)] and the phase $\phi[$ Fig. 2(d)] of the transmission through a finite size structure inserted horizontally between two wave guides [25] [see the inset of Fig. 2(c)]. Indeed, writing the transmission coefficient $\operatorname{tr}=|\operatorname{tr}| e^{j \phi}$ under the form $\operatorname{tr}=e^{j k L}$, where $L$ is the total length of the finite structure, one obtains the effective wave number $k=\phi / L-j \ln |\operatorname{tr}| / L$. One can notice that despite the small number of cells $(N=4)$ used in the periodic structure, the amplitude and the phase describe very well the band structure of the infinite system $(N \rightarrow \infty)$ [Fig. 2(a)]. As mentioned above, because of the periodicity of the band gap structure, we limited ourselves to the reduced frequency region $0 \leqslant \Omega \leqslant \pi$.

Inside the first gap $k D=\pi \pm j \kappa$ and the dispersion relation [Eq. (24)] becomes

$$
\cosh (\kappa / 2)=\frac{3}{2 \sqrt{2}} \sin (\Omega) .
$$

Equation (25) gives the imaginary part $\kappa$ of the reduced wave vector $k D$ inside the gaps which is responsible for the attenuation of the modes that may lie inside these gaps when a defect is inserted in the structure such as the surface $[21,22]$. From the above results, one can deduce that the center of the first gap is given by $\Omega=\pi / 2$ and the value of $\kappa$ at this frequency is $\kappa \simeq 0.69$ [Eq. (25)] as illustrated by the dashed curves in Figs. 2(a) and 2(b).

The theoretical transmission curves (amplitude and phase) are obtained from Eq. (15), whereas the transmission measurements have been realized by using the tracking generator coupled to a spectrum analyzer in the frequency range of 10 to $100 \mathrm{MHz}$. The attenuation inside the coaxial cables was simulated by introducing a complex dielectric constant $\varepsilon\left(\varepsilon=\varepsilon^{\prime}-j \varepsilon^{\prime \prime}\right)$. The attenuation coefficient $\alpha^{\prime \prime}$ can be expressed as $\alpha^{\prime \prime}=\varepsilon^{\prime \prime} \omega / c$. On the other hand, the attenuation specification data supplied by the manufacturer of the coaxial cables in the frequency range of 10 to $100 \mathrm{MHz}$ can be approximately fitted with the expression $\ln \left(\alpha^{\prime \prime}\right)=\gamma+\delta \ln (\omega)$, where $\gamma$ and $\delta$ are two constants. From this fitting procedure, a useful expression for $\varepsilon^{\prime \prime}$ as a function of frequency can be obtained under the form $\varepsilon^{\prime \prime}=0.017 f^{-0.5}$, where the frequency $f$ is expressed in Hz. The experimental results are very well fitted by the 1D model using the Green's function method. One can notice in Fig. 2(c) that the attenuation inside the cables induces transmission depletion especially at high frequencies.

As concerns the eigenmodes of the finite SL, one can distinguish, as described in Sec. II B, the bulk modes [Eq. (18)] lying inside the allowed bands and the surface modes [Eq. (22)] lying inside the forbidden bands. The expression giving the surface modes [Eq. (22)] can be written as [18]

$$
Z_{A} C_{A} S_{B}+Z_{B} C_{B} S_{A}=0
$$

together with the condition [Eq. (21)]

$$
\left|C_{A} C_{B}-\frac{Z_{B}}{Z_{A}} S_{A} S_{B}\right|<1
$$

when the structure is terminated by a segment and 


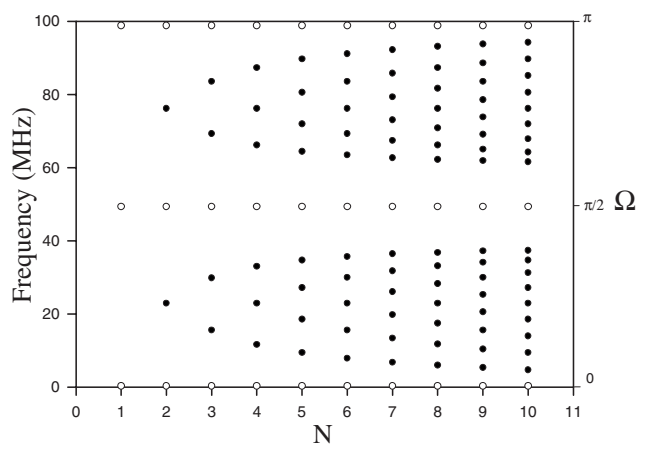

FIG. 3. Variation of the eigenmodes of the finite SL as a function of the number of cells $N$. Open and solid circles have the same meaning as in Fig. 2.

$$
\left|C_{A} C_{B}-\frac{Z_{A}}{Z_{B}} S_{A} S_{B}\right|<1
$$

when the structure is terminated by a loop.

In the particular case considered here, $C_{A}=C_{B}=\cos (\Omega)$ and $S_{A}=S_{B}=\sin (\Omega)$. Therefore, Eq. (26) becomes simply

$$
\sin (2 \Omega)=0, \quad \text { i.e., } \Omega=n \pi / 2,
$$

where $n$ is an integer. If $n$ is even (i.e., $\Omega=0, \pi, 2 \pi, \ldots$ ), then neither Eq. (27) nor Eq. (28) are fulfilled since the left-hand term in these equations is unity. As mentioned above, this situation corresponds to the center of the Brillouin zone [i.e., $k D=0$, see Eq. (24)] where the band gaps close. However, if $n$ is odd (i.e., $\Omega=\pi / 2,3 \pi / 2, \ldots$ ), then only Eq. (27) is fulfilled since $Z_{B}<Z_{A}$, which means that all the surface modes appear on the surface of the structure terminated by a segment and no surface modes appear when the structure terminates with a loop. In Fig. 2(a) we have plotted by open circles the surface mode lying in the first gap at $\Omega=\pi / 2$ (i.e., $f=49.34 \mathrm{MHz}$ ) as well as the frequencies lying at the band gap edges $\Omega=0$ and $\Omega=\pi$ (i.e., $f=0$ and $f=98.86 \mathrm{MHz}$ ). These modes, given by Eq. (29), are independent of $N$. However, as predicted, there exist $N-1=3$ modes in each band given by Eq. (18).

In order to give experimental evidence of the eigenmodes of the finite SL, we measure the transmission coefficient through the structure grafted vertically along the guide [see the inset of Fig. 2(e)]. Indeed, from Eqs. (14) and (16), one can notice that the maxima of the transmission (i.e., $\operatorname{tr}_{v}=1$ ) occur at the frequencies of the finite SL with vanishing magnetic field on both ends. An example of the transmission spectrum of a finite structure made of four loops is sketched in Fig. 2(e). The frequencies of the corresponding maxima are reported in Fig. 2(b). One can notice that the positions of the eigenmodes of the finite SL coincide exactly with those obtained from theory [Fig. 2(a)].

Figure 3 shows the variation of the eigenmodes of a finite $\mathrm{SL}$ as a function of the number of cells $N$. For $N=1$ (one cell), the eigenmodes are given by Eq. (29) and we can distinguish the modes lying at the closing of the band gaps (i.e., $\Omega=0$ and $\Omega=\pi$ ) and the surface mode lying at the center of the band gap (i.e., $\Omega=\pi / 2$ ). When $N$ increases, the above

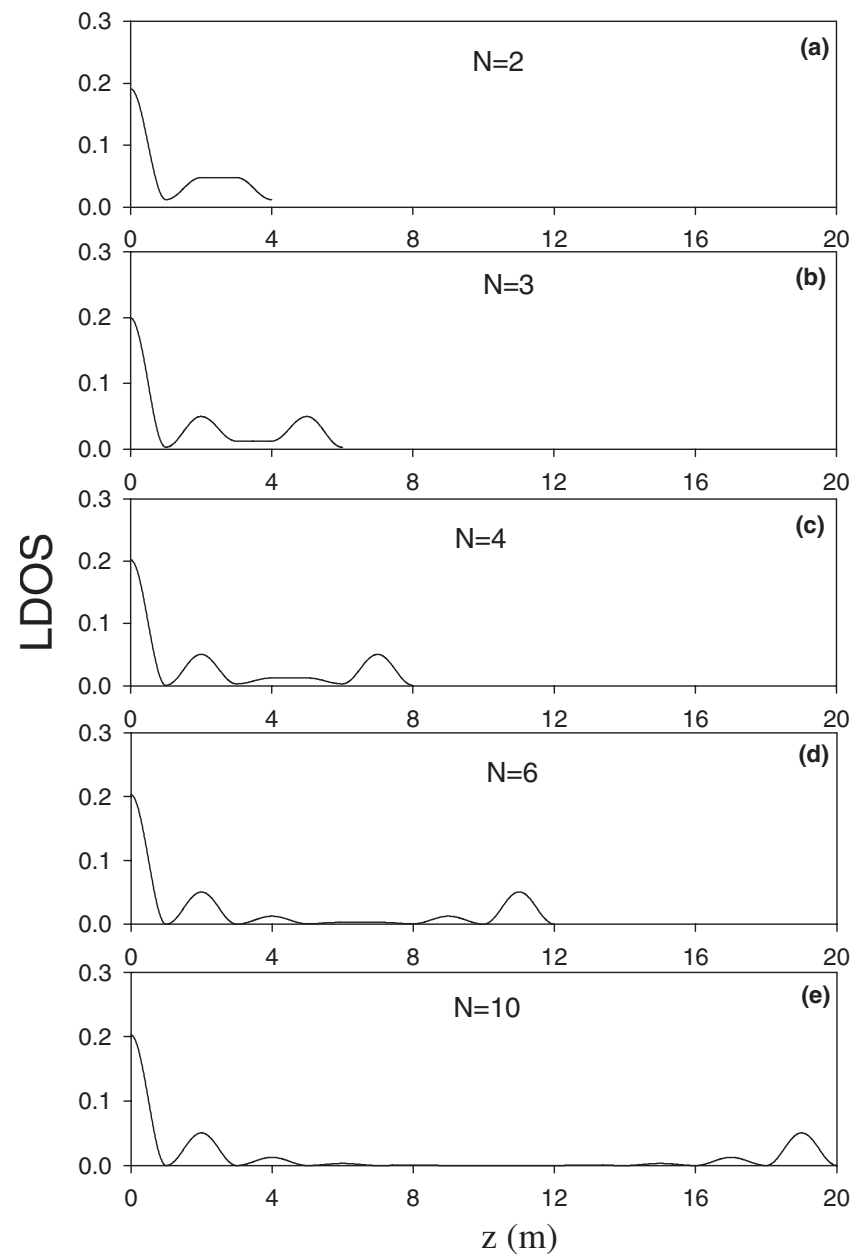

FIG. 4. The local density of states (LDOS) (in arbitrary units) as a function of the space position $z$ for the mode lying at the central gap frequency $\Omega=\pi / 2$ (Fig. 3) for $N=2$ (a), 3 (b), 4 (c), 6 (d), and 10 (e). The finite SL is terminated by a segment and a loop at the left and the right of the structure, respectively.

modes remain constant, whereas there exists $N-1$ modes in each band for every value of $N$ in accordance with the analytical results in Sec. II B.

The Green's function approach enables one also to deduce the local and total densities of states (LDOS). The details of these calculations are given in Refs. [18,22,36,37]. The LDOS reflects the behavior of the square modulus of the electric field inside the structure. An analysis of the LDOS as a function of the space position (Fig. 4) shows, as expected, that the surface mode lying in the first gap at $\Omega=\pi / 2$ exhibits a strong localization at the surface terminated with a segment, with almost the same localization length regardless of the length of the structure. Indeed, the localization length is defined as $l=D /|\kappa|$, where $\kappa$ is the imaginary part of the reduced wave vector $k D$ [Eq. (25)]. As mentioned in Sec. III $A, \kappa \simeq 0.69$ for the surface mode lying at $\Omega=\pi / 2$ and therefore $l \simeq 3 \mathrm{~m}$ which is in accordance with the results of Fig. 4.

\section{B. Case of a finite structure with symmetric cells}

In what follows, we consider a finite periodic structure made of symmetric cells. Each cell is composed of a sym- 

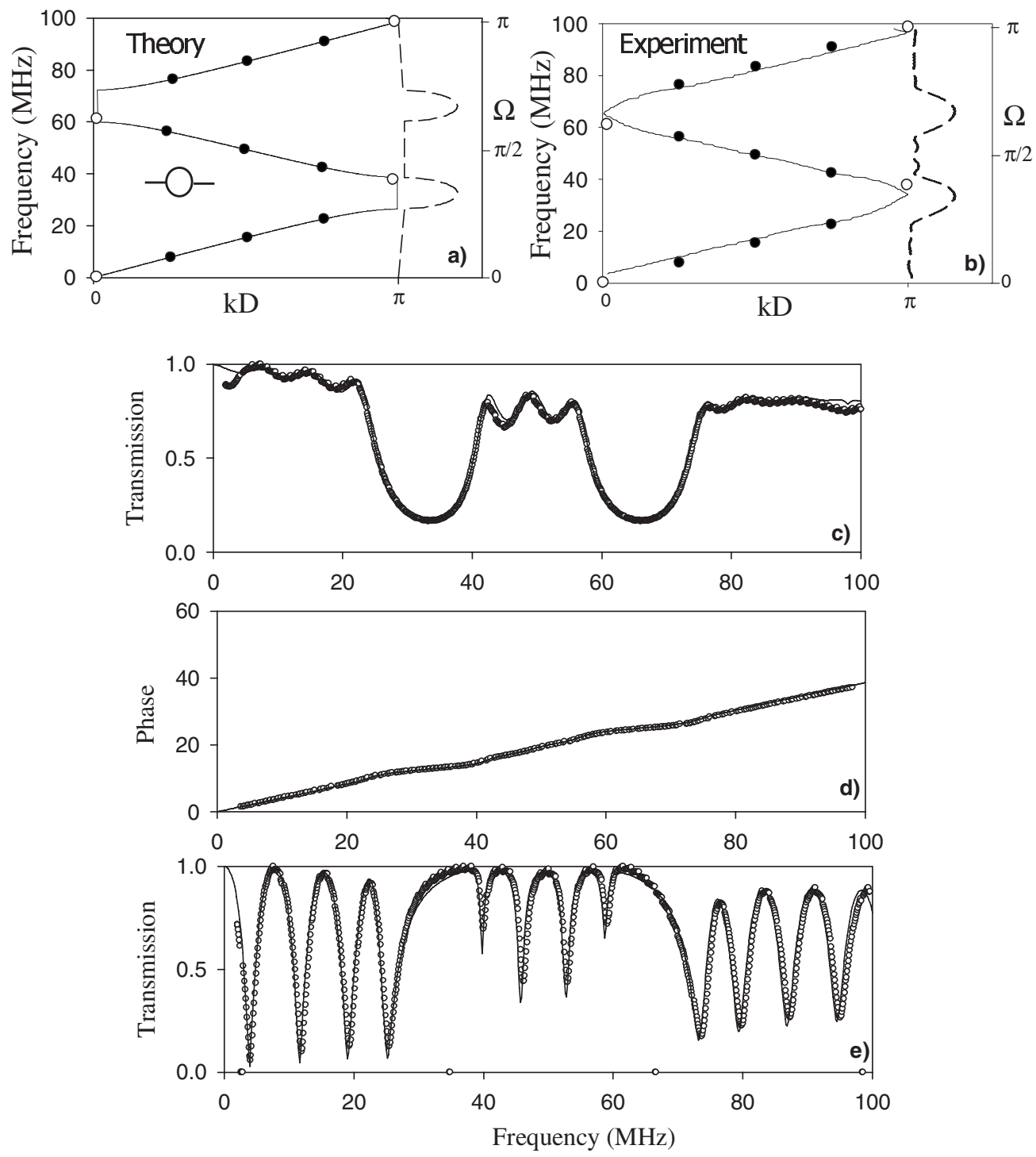

FIG. 5. Same as in Fig. 2 but here each cell is made of a loop sandwiched between two identical segments [symmetric cell, see the inset of Fig. 5(a)].

metric loop of type $B$ inserted between two identical segments of type $A$. Therefore, each cell becomes equivalent to a $50 \Omega / 25 \Omega / 50 \Omega$ trisegment [see the inset of Fig. 5(a)]. One can notice that the two surfaces surrounding the cell are equivalent and therefore the cell is symmetrical. Also, when connected together, the cells give rise to a finite SL made of loops separated by segments of length $2 \mathrm{~m}$ and the whole structure terminates by segments of length $1 \mathrm{~m}$ on both sides.

In this case, the dispersion relation (7) becomes [18]

$$
\cos (k D)=C_{A}^{2} C_{B}-C_{B}^{2} C_{A}-C_{A} S_{A} S_{B}\left(Z_{A} / Z_{B}+Z_{B} / Z_{A}\right),
$$

where $D=2 d_{A}+d_{B}$. In the particular case where $d=d_{A}=d_{B}$ $=1 \mathrm{~m}$ and $Z_{A} / Z_{B}=2$, the above equation becomes simply

$$
\cos (k D)=\frac{\cos (\Omega)}{2}\left[9 \cos ^{2}(\Omega)-7\right] \text {. }
$$

The band gap edges are given by $\cos (k D)= \pm 1$, namely, $\cos (\Omega)= \pm 1, \pm 1 / 3$, and $\pm 2 / 3$. Therefore,

$$
\Omega=0,0.27 \pi, 0.39 \pi, 0.61 \pi, 0.73 \pi, \pi, \ldots .
$$

Inside the two first gaps $k D=\pi \pm j \kappa$ and $k D= \pm j \kappa$, respectively, and the dispersion relation (31) becomes

$$
\cosh (\kappa)=\mp \frac{\cos (\Omega)}{2}\left[9 \cos ^{2}(\Omega)-7\right] \text {. }
$$

Thus, one can deduce the reduced frequencies at the center of the first two gaps, namely,

$$
\cos (\Omega)=\frac{7 \sqrt{7}}{9 \sqrt{3}}, \text { i.e., } \Omega \simeq 0.33 \pi \text { and } \Omega \simeq 0.67 \pi
$$

as well as, the corresponding values of $\kappa[\kappa \simeq 0.59$, see the dashed curves in Fig. 5(a)]. The surface modes [Eq. (22)] are given in general by [18] 


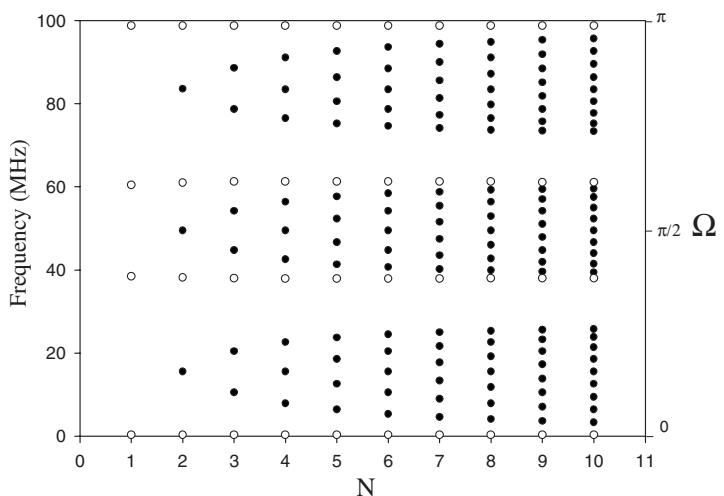

FIG. 6. Variation of the eigenmodes of the finite SL as a function of the number of cells $N$ for the structure described in Fig. 5.

$$
2 C_{A} S_{A} C_{B}+S_{B}\left(C_{A}^{2} \frac{Z_{A}}{Z_{B}}+S_{A}^{2} \frac{Z_{B}}{Z_{A}}\right)=0 .
$$

In the particular case considered here, $C_{A}=C_{B}=\cos (\Omega)$, $S_{A}=S_{B}=\sin (\Omega)$, and $Z_{A} / Z_{B}=2$. Then, Eq. (35) becomes

$$
\sin (\Omega)\left[9 \cos ^{2}(\Omega)-1\right]=0,
$$

which leads to

$$
\sin (\Omega)=0 \text {, i.e., } \Omega=0, \pi, 2 \pi
$$

or

$\cos (\Omega)= \pm 1 / 3$, i.e., $\Omega=0.39 \pi, 0.61 \pi, 1.39 \pi, 1.61 \pi, \ldots$.

However, the two latter equations give $\cos (k D)= \pm 1[\mathrm{Eq}$. (31)]. Consequently, as mentioned in Sec. II B, the finite periodic SL with symmetric cells does not exhibit surface modes inside the band gaps, but leads only to constant frequency band-edge modes. These results are similar to those found by Ren $[34,35]$ for a complete confinement of electronic states in finite one-dimensional systems. Of course, in addition to the band-edge modes, one can expect $N-1$ modes in each band given by Eq. (18).

Figure 5(a) summarizes the numerical results corresponding to the analytical results detailed above, whereas Fig. 5(b) together with Figs. 5(c)-5(e) summarize the experimental results. One can notice a good agreement between theory and experiment concerning the band gap structure as well as the eigenmodes of the finite SL. Among the different eigenmodes, one can distinguish the band-edge modes plotted by open circles and the bulk band modes $(N-1=3)$ lying inside each allowed band. Figure 6 gives the variation of the eigenmodes of the finite SL as a function of the number of cells $N$. One can notice the existence of band-edge modes lying at a constant frequency independent of $N$ [Eqs. (37) and (38)] and $N-1$ modes in each band and for each value of $N$.

\section{CONCLUSION}

In this paper, we have presented theoretical and experimental evidence of the existence of two types of modes in finite size 1D coaxial photonic crystals made of a periodic repetition of $N$ cells. In particular, we have shown the existence of $N-1$ modes that match the bulk bands and one additional mode per gap if the cell is asymmetric. However, if the cell is symmetric, the additional mode falls at a constant frequency at the bulk band edges. These modes are independent of $N$. These results generalize our previous findings $[18,21,22]$ on the existence of surface modes associated to two semi-infinite complementary SLs obtained from the cleavage of an infinite SL between two cells. The theoretical results are confirmed experimentally by using asymmetric cells made of a simple coaxial cable connected to a loop (constituted of two identical coaxial cables) and symmetric cells made of a loop inserted between two identical coaxial cables. The band gap structure and the different eigenmodes of these photonic systems are obtained, respectively, from the measurement of the transmission coefficient through a finite size structure inserted horizontally between two waveguide cables and a finite size structure grafted vertically along a guide. The experimental results are in good agreement with theoretical calculations based on the formalism of the Green's function. Finally, let us mention that the results obtained here remain also valid for other physical situations. First, as mentioned in the Introduction, the same rules apply for coaxial PCs if the boundary condition at the ends of the finite structure is the vanishing of the electric field instead of the magnetic field. On the other hand, for elastic waves in SLs, we obtained the same rule for the two following cases: (i) transverse elastic waves in finite size solid-solid SL [18] and (ii) sagittal elastic waves in finite size solid-fluid SL [38]. In both cases, the boundary conditions at the ends of the structure are free of stress.

Note added in proof: Recently, we noticed that similar results to those presented here for coaxial photonic crystal have been obtained theoretically in a different demonstration for transverse elastic waves in 1D phononic crystal [39]. We believe that similar conclusions can be derived for phonons in a linear multi-atomic chain, i.e., when the unit cell is constituted by several atoms differing either by their mass or the spring constant that link them together. Actually, this is straightforward in the simple cases of a mono-atomic and bi-atomic linear chain. Recent experiments [40] on linear chains composed of welded steel spheres of the same or different diameters have been explained in this framework.

\section{ACKNOWLEDGMENTS}

E.E. and Y.E. gratefully acknowledge the hospitality of Institut d'Électronique, de Microélectronique et de Nanotechnologie (IEMN) and UFR de Physique, Université de Lille 1. This work was supported by "le Fond Européen de Développement Régional” (FEDER), INTERREG III France-Wallonie-Flandre (PREMIO), and "le Conseil Régional Nord-Pas de Calais." The authors would like to thank Professor Akjouj for the experimental results. 
[1] E. Yablonovitch, Phys. Rev. Lett. 58, 2059 (1987).

[2] S. John, Phys. Rev. Lett. 58, 2486 (1987).

[3] See, for example, J. D. Joannopoulos, R. D. Meade, and J. N. Winn, Photonic Crystals (Princeton University Press, Princeton, NJ, 1995); Photonic Band Gap and Localization, edited by C. M. Soukoulis (Plenum, New York, 1993); Photonic Band Gap Materials, edited by C. M. Soukoulis (Kluwer, Dordrecht, 1996).

[4] S. Johnson and J. D. Joannopoulos, Photonic Crystals: The Road from Theory to Practice (Kluwer Academic Publishers, Boston, 2002).

[5] P. Yeh, Optical Waves in Layered Media (Wiley, New York, 1988).

[6] A. A. Bulgakov and V. R. Koturn, Opt. Spectrosc. 56, 269 (1984).

[7] W. M. Robertson and M. S. May, Appl. Phys. Lett. 74, 1800 (1999).

[8] F. Ramos-Mendieta and P. Halevi, J. Opt. Soc. Am. B 14, 370 (1997).

[9] M. L. H. Lahlaouti, A. Akjouj, B. Djafari-Rouhani, L. Dobrzynski, M. Hammouchi, E. H. El Boudouti, A. Nougaoui, and B. Kharbouch, Phys. Rev. B 63, 035312 (2001).

[10] F. Villa, J. A. Gaspar-Armenta, and F. Ramos-Mendieta, Opt. Commun. 216, 361 (2003).

[11] Y. El Hassouani, E. H. El Boudouti, H. Aynaou, B. DjafariRouhani, and V. R. Velasco, J. Opt. A, Pure Appl. Opt. 9, 308 (2007).

[12] D. N. Chigrin, A. V. Lavrinenko, D. A. Yarotsky, and V. Gaponenko, J. Lightwave Technol. 17, 2018 (1999).

[13] T. Okamoto, T. Kamiyama, and I. Yamaguchi, Opt. Lett. 18, 1570 (1993).

[14] W. M. Robertson, J. Lightwave Technol. 17, 2013 (1999).

[15] M. Shinn and W. M. Robertson, Sens. Actuators B 105, 360 (2005)

[16] J. A. Gaspar-Armenta and F. Villa, J. Opt. Soc. Am. B 21, 405 (2004).

[17] M. L. H. Lahlaouti, A. Akjouj, B. Djafari-Rouhani, and L. Dobrzynski, Phys. Rev. B 61, 2059 (2000).

[18] E. H. El Boudouti, B. Djafari-Rouhani, E. M. Khourdifi, and L. Dobrzynski, Phys. Rev. B 48, 10987 (1993); E. H. El Boudouti, B. Djafari-Rouhani, A. Akjouj, and L. Dobrzynski, ibid. 54, 14728 (1996).

[19] W. Chen, Y. Lu, H. J. Maris, and G. Xiao, Phys. Rev. B 50, 14506 (1994)

[20] N-W. Pu and J. Bokor, Phys. Rev. Lett. 91, 076101 (2003);
N-W. Pu, Phys. Rev. B 72, 115428 (2005).

[21] B. Djafari-Rouhani, E. H. El Boudouti, A. Akjouj, L. Dobrzynski, J. O. Vasseur, A. Mir, N. Fettouhi, and J. Zemmouri, Vacuum 63, 177 (2001).

[22] Y. El Hassouani, H. Aynaou, E. H. El Boudouti, B. DjafariRouhani, A. Akjouj, and V. R. Velasco, Phys. Rev. B 74, 035314 (2006).

[23] G. J. Schneider, S. Hanna, J. L. Davis, and G. H. Watson, J. Appl. Phys. 90, 2642 (2001).

[24] L. Poirier and A. Haché, Appl. Phys. Lett. 78, 2626 (2001).

[25] E. H. El Boudouti, N. Fettouhi, A. Akjouj, B. Djafari-Rouhani, A. Mir, J. O. Vasseur, L. Dobrzynski, and J. Zemmouri, J. Appl. Phys. 95, 1102 (2004).

[26] A. Haché and L. Poirier, Phys. Rev. E 65, 036608 (2002); J. N. Munday and W. M. Robertson, Appl. Phys. Lett. 80, 518 (2002).

[27] H. Aynaou, E. H. El Boudouti, Y. El Hassouani, A. Akjouj, B. Djafari-Rouhani, J. Vasseur, A. Benomar, and V. R. Velasco, Phys. Rev. E 72, 056601 (2005).

[28] E. L. Albuquerque and M. G. Cottam, Phys. Rep. 376, 225 (2003), and references therein

[29] L. Dobrzynski, Surf. Sci. Rep. 11, 139 (1990).

[30] J. Vasseur, A. Akjouj, B. Djafari-Rouhani, L. Dobrzynski, and E. H. El Boudouti, Surf. Sci. Rep. 51, 1 (2004).

[31] M. del Mar Sanchez-Lopez, J. A. Davis, and K. Crabtree, Am. J. Phys. 71, 1314 (2003).

[32] A. Haché and A. Slimani, Am. J. Phys. 72, 916 (2004).

[33] J. A. Davis, D. A. Miller, M. del Mar Sanchez-Lopez, and J. Cos, Am. J. Phys. 74, 1066 (2006).

[34] S. Y. Ren, Phys. Rev. B 64, 035322 (2001).

[35] S. Y. Ren, Ann. Phys. (N.Y.) 301, 22 (2002).

[36] E. H. El Boudouti and B. Djafari-Rouhani, Phys. Rev. B 49, 4586 (1994); M. Hammouchi, E. H. El Boudouti, A. Nougaoui, B. Djafari-Rouhani, M. L. H. Lahlaouti, A. Akjouj, and L. Dobrzynski, ibid. 59, 1999 (1999).

[37] M. L. Lahlaouti, A. Akjouj, B. Djafari-Rouhani, L. Dobrzynski, M Hammouchi, E. H. El Boudouti, and A. Nougaoui, J. Opt. Soc. Am. A 16, 7 (1999).

[38] Y. El Hassouani, E. H. El Boudouti, B. Djafari-Rouhani, H. Aynaou, and L. Dobrzynski, Phys. Rev. B 74, 144306 (2006).

[39] S.-Y. Ren and Y.-C. Chang, Phys. Rev. B 75, 212301 (2007).

[40] A-C. Hladky, A. Devos, and M. de Billy, J. Acoust. Soc. Am. 116, 117 (2004); A.-C. Hladky, G. Allan, and M. de Billy, J. Appl. Phys. 98, 054909 (2005). 